\title{
Electron-correlation effects in appearance-potential spectra of $\mathrm{Ni}$
}

\author{
M. Potthoff,. 7 T. Wegner, and W. Nolting \\ Lehrstuhl Festkörpertheorie, Institut für Physik, Humboldt-Universität zu Berlin, 10115 Berlin, Germany \\ T. Schlathölter \\ Division Technical Systems, Philips Research, 22335 Hamburg, Germany \\ M. Vonbank and K. Ertl \\ Max-Planck-Institut für Plasmaphysik, EURATOM Association, 85740 Garching bei München, Germany \\ J. Braun and M. Donath \\ Physikalisches Institut, Universität Münster, 48149 Münster, Germany
}

\begin{abstract}
Spin-resolved and temperature-dependent appearance-potential spectra of ferromagnetic Nickel are measured and analyzed theoretically. The Lander self-convolution model which relates the line shape to the unoccupied part of the local density of states turns out to be insufficient. Electron correlations and orbitally resolved transition-matrix elements are shown to be essential for a quantitative agreement between experiment and theory.
\end{abstract}

\section{INTRODUCTION}

Due to the Coulomb interaction, an electron approaching a metal surface can excite a core electron into a state of the unoccupied bands. Above a threshold energy both, the excited core electron and the de-excited primary electron are scattered into valence states just above the Fermi energy. The appearance-potential spectroscopy (APS) 1 , 3 . ationless transition as a function of the energy of the primary electrons by detecting the emitted X-rays or Auger electrons of the subsequent core-hole decay. Since corehole formation is involved, the method is element specific and local. Its comparatively simple experimental setup and its surface sensitivity qualifies APS as a useful technique for surface analysis. For a ferromagnetic material, the spin dependence of the AP signal obtained by using a polarized electron beam reflects the surface magnetization as has heendemonstrated for the transition metals Fe and Ni.6.6. 6 In a sense APS can be considered to be complementary to Auger-electron spectroscopy (AES). 10 While the Auger line shape resulting from core-valencevalence (CVV) transitions yields information on the occupied part of the valence band, APS is sensitive to the unoccupied electronic structure. Contrary to $\mathbf{k}$ resolved (inverse) photoemission, the AP transition is more or less localized in real space. This suggests to employ APS as a quantitative probe for the unoccupied part of the (spindependent) local valence density of states (DOS).

In most cases the interpretation of appearancepotential spectra is still based on a simple independentelectron model suggested by Lander in 1953:11 Hereafter, the line shape is given by the self-convolution of the unoccupied part of the DOS. The desired information can then be obtained by de-convolution techniques 12,13 Within the context of AES, however, it soon became clear that the self-convolution model seems to be oversimplified. Powell14 discovered an "anomalous" shape of the CVV Auger line of Ag which has been attributed to electron-correlation effects. Correlations may be significant also for APS because of the direct interaction of the two additional valence electrons in the final state This is demonstrated by the Cini-Sawatzky theory:15. 16 Within the framework of the single-band Hubbard model, the two-particle (APS/AES) excitation spectrum is dominated by a pronounced satellite feature which is split off the band-like part by a characteristic energy of the order of the on-site Coulomb interaction $U$. For more realistic (multi-band) Hubbard models the direct eqrrelations give

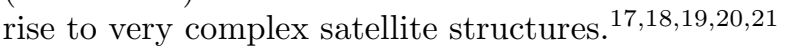

A second shortcoming of the Lander model consists in the fact that transition-matrix elements are not taken into account. Modern theories for APS (AES) based on density-functional theory and the local-density imation (LDA) have overcome this deficiency.22.23 Although the direct correlations are still neglected in these approaches, it seems that the $\mathrm{AP}$ spectra of $\mathrm{Fe}$ and $\mathrm{Ni}$, for example, are well understood 23.24 - serious indications for correlation effects have not been observed. This is surprising since it is well known that the Auger line shape of $\mathrm{Ni}$ cannot be explained within an effective independentelectron model 25 It is dominated by a strong spectralweight transfer due to an on-site interaction of the order of $U \approx 2-3 \mathrm{eV}$ to he compared with the effective $d$-band width $\Delta \approx 3 \mathrm{eV}$. 102

Clearly, the question what really determines the appearance-potential line shape of transition metals, can only be answered a posteriori - namely by comparison with a theory that realistically includes electroncorrelation as well as matrix-element effects, orbital degeneracy and $s p$ - $d$ hybridization from the very beginning. The analysis of the AP line shape of $\mathrm{Ni}$, as a prototypical ferromagnetic $3 d$ metal, is the purpose of the present paper. In the experiment spin-resolved spectra from excitation of the $2 p_{3 / 2}\left(L_{\mathrm{III}}\right)$ core level are recorded for different temperatures ranging from $T=100 \mathrm{~K}$ up to 
and slightly above the Ni Curie point $T_{\mathrm{C}}=624 \mathrm{~K} .27$ The theoretical interpretation of the line shape is essentially based on a multi-band Hubbard-type model with general on-site Coulomb interaction and a realistic parameterization of the (LDA) one-particle electronic structure as an input for a subsequent many-body calculation. Standard diagrammatic techniques are used to account for correlations. The transition-matrix elements are calculated within the usual intra-atomic approximation. The novel feature of the approach is that it allows to study separately the DOS effect (bare self-convolution), the temperature-dependent effect of the (direct and remaining indirect) correlations as well as the effect of the transition-matrix elements.

\section{EXPERIMENT}

Experiments are performed for a Nifitp) singlecrystal surface with in-plane magnetization. 028 The spinpolarized electron beam used for excitation is emitted from a GaAs photocathode irradiated by circularly polarized light. The longitudinal spin polarization of the emitted photoelectrons is changed into transversal by $90^{\circ}$ electrostatic deflection. To correct for the incomplete polarization of the electrons $(P \approx 30 \%)$, all data have been rescaled to a $100 \%$ hypothetical beam polarization and alignment between the electron-polarization and the sample-magnetization vector. In the present setup the core-hole decay is detected via soft-X-ray emission (SXAPS). The detector arrangement consists of a multichannel plate with filters and a CsI layer acting as photon-to-electron converter. The APS signal is measured as a function of the primary energy of the electrons. Modulation of the sample potential together with lock-in technique is employed to separate the signal from the otherwise overwhelming background. For the potential modulation a peak-to-peak voltage of $2 \mathrm{~V}$ has been chosen. This value ensures high APS signals for the $2 p_{3 / 2}$ core level without modulation-induced broadening effects. Further details of the experimental setup and the sample preparation have been described elsewhere.

Fig. 1 shows the differential AP intensity as a function of the energy of primary electrons with polarization parallel (minority, $\downarrow$ ) or antiparallel (majority, $\uparrow$ ) to the target magnetization. The displayed energy range covers the emission from the $L_{\mathrm{III}}$ transition. The $L_{\mathrm{II}}$ emission would be visible at higher energies shifted by the $2 p$ spinorbit splitting of about $17.2 \mathrm{eV}$.

For $T / T_{\mathrm{C}}=0.16$ the system is close to ferromagnetic saturation. The appearance-potential spectrum shows a strongly asymmetric intensity ratio as well as a spin splitting of the main peak at $E=852.3 \mathrm{eV}$ (dotted line). Since $\mathrm{Ni}$ is a strong ferromagnet, there are only few unoccupied $d$ states available in the majority spin channel, and thus $I_{\uparrow}<I_{\downarrow}$ holds for the (non-differential) intensities. This is the dominant spin effect. The intensity asymmetry in the main peak gradually diminishes with

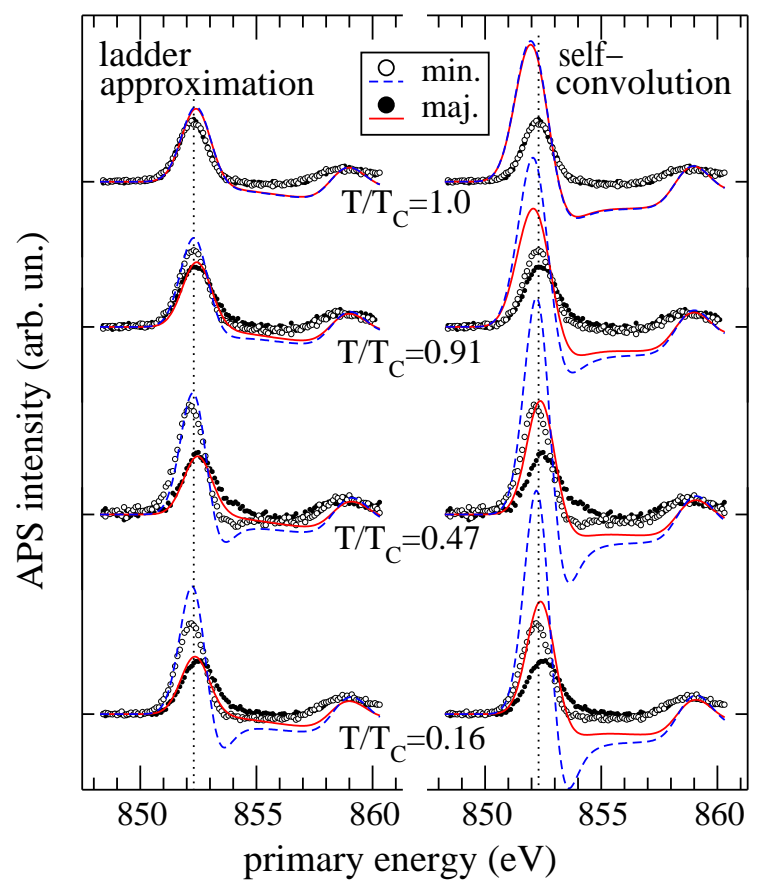

FIG. 1: Spin-resolved $L_{\text {III }} \mathrm{Ni}$ appearance-potential spectrum for different temperatures $T / T_{\mathrm{C}}$. Data points: measured differential intensity $d I / d E$ as a function of the primary energy. For better comparison with theory the same data are shown twice (left and right panel). Lines, left: theory with direct and indirect correlations included (ladder approximation). Lines, right: indirect correlations included only (self-convolution).

increasing temperature and vanishes at $T_{\mathrm{C}}$. Note that only the position of the majority peak shifts with $T$ while the minority peak position remains unchanged.

The main peak is related to the high density of states at the Fermi energy and is mainly due to $d-d$ character of the two final-state electrons. Additional small $s$ - $d$ contributions are present in the secondary peak at $E \approx 859 \mathrm{eV}$ as has been conchided from the analysis of the transitionmatrix elements.6. 23 This structure has been identified as originating from a DOS discontinuity deriving from the $L_{7}$ critical point in the Brillouin zone. 6 No temperature dependence and spin asymmetry is detectable here.

\section{THEORY}

The theoretical approach is based on the usual twostep description assuming the lifetime of the core hole to be sufficiently long. 129 The AP line shape is then unaffected by the cross section for the core-hole decay step but solely determined by the excitation step. According to Fermi's golden rule, the intensity can be written as:

$$
\begin{aligned}
& I_{\sigma_{c} \sigma_{i}}\left(\mathbf{k}_{\|}, E\right) \propto \operatorname{Im} \sum_{L_{1} L_{2} L_{1}^{\prime} L_{2}^{\prime}} M_{L_{1} L_{2}}^{\sigma_{c} \sigma_{i}}\left(\mathbf{k}_{\|}, E\right) \times \\
& \quad\left\langle\left\langle c_{i L_{1} \sigma_{c}} c_{i L_{2} \sigma_{i}} ; c_{i L_{2}^{\prime} \sigma_{i}}^{\dagger} c_{i L_{1}^{\prime} \sigma_{c}}^{\dagger}\right\rangle\right\rangle_{E}\left(M_{L_{1}^{\prime} L_{2}^{\prime}}^{\sigma_{c} \sigma_{i}}\left(\mathbf{k}_{\|}, E\right)\right)^{*} .
\end{aligned}
$$


For an incoming electron with spin $\sigma_{i}=\uparrow, \downarrow$, one has to consider the "singlet" transition, i. e. excitation of a core electron with spin $\sigma_{c}=-\sigma_{i}$, as well as the "triplet" transition with $\sigma_{c}=\sigma_{i}$. Since the spin state of the final core hole is not detected, the intensities have to be summed incoherently:

$$
I_{\sigma_{i}}\left(\mathbf{k}_{\|}, E\right) \equiv I_{\sigma_{c} \sigma_{i}}\left(\mathbf{k}_{\|}, E\right)+I_{-\sigma_{c} \sigma_{i}}\left(\mathbf{k}_{\|}, E\right) .
$$

Above the Curie temperature $I_{\sigma_{i}}=I_{-\sigma_{i}}$.

The "raw spectrum" resulting from an intra-atomic transition at the lattice site $i$ is given by the imaginary part of the retarded two-particle (Zubarev30) Green function in Eq. (1). It describes the correlated propagation of the two additional final-state electrons at energy $E$ :

$$
\begin{aligned}
& \left\langle\left\langle c_{i L_{1} \sigma_{c}} c_{i L_{2} \sigma_{i}} ; c_{i L_{2}^{\prime} \sigma_{i}}^{\dagger} c_{i L_{1}^{\prime} \sigma_{c}}^{\dagger}\right\rangle\right\rangle_{E}= \\
& -\int_{0}^{\infty} d t e^{i E t}\left\langle\left[c_{i L_{1} \sigma_{c}}(t) c_{i L_{2} \sigma_{i}}(t) ; c_{i L_{2}^{\prime} \sigma_{i}}^{\dagger}(0) c_{i L_{1}^{\prime} \sigma_{c}}^{\dagger}(0)\right]_{-}\right\rangle .
\end{aligned}
$$

Here $\langle\cdots\rangle$ is the thermodynamical average, $[\ldots, \ldots]_{-}$the commutator, and $\mathcal{O}(t)=\exp (i H t) \mathcal{O} \exp (-i H t)$ is the Heisenberg time dependence of an operator $\mathcal{O}$. The Green function is expressed in terms of annihilators (creators) $c_{i L \sigma}^{(\dagger)}$ which refer to a tight-binding one-particle basis. Due to translational symmetry of the fcc lattice the $i$ dependence is only formal. $L=\{l, m\}$ characterizes the angular momentum of the localized $3 d, 4 s$, and $4 p$ basis functions. In total there are $2 \times 9$ orbitals $|i L \sigma\rangle=c_{i L \sigma}^{\dagger} \mid$ vac. $\rangle$ per site.

Because of the definite $L$ character of the basis orbitals, the orbitals on different sites are mutually nonorthogonal: $\left\langle i L \sigma \mid i^{\prime} L^{\prime} \sigma^{\prime}\right\rangle=S_{i i^{\prime}}^{L L^{\prime}} \delta_{\sigma \sigma^{\prime}}$. There are different advantages to develop the many-body formalism for a non-trivial overlap matrix $\mathbf{S} \neq \mathbf{1}$ (see Refs. 31, 32): A unique decomposition of the spectrum into $s, p$, and $d$ parts is possible, the basis orbitals are more localized, 31 and in the interaction part of the Hamiltonian the on-site Coulomb interaction among the $3 d$ electrons can be dealt with separately. Furthermore, the same basis states enter the definition of the transition-matrix elements, and their definite angular-momentum character implies helpful selection rules.

The transition-matrix elements in Eq. (11),

$$
\begin{aligned}
& M_{L_{1} L_{2}}^{\sigma_{c} \sigma_{i}}\left(\mathbf{k}_{\|}, E\right)= \\
& { }^{(1)}\left\langle 2 \mathrm{p},\left.\sigma_{c}\right|^{(2)}\left\langle\mathbf{k}_{\|} E \sigma_{i}\left|\frac{1}{\left|\mathbf{r}_{1}-\mathbf{r}_{2}\right|}\right| i L_{1} \sigma_{c}\right\rangle^{(1)} \mid i L_{2} \sigma_{i}\right\rangle^{(2)},
\end{aligned}
$$

are calculated byassuming the transition to be intraatomic as usual.1022,23 The different wave functions as well as the Coulomb operator $1 /\left|\mathbf{r}_{1}-\mathbf{r}_{2}\right|$ are expanded into spherical harmonics, the angular integrations are performed analytically, and the numerical radial integrations are cut at the Wigner-Seitz radius (see Ref. 33 for details).
Surface effects enter the theory via the high-energy scattering state $\left|\mathbf{k}_{\|} E \sigma_{i}\right\rangle$. It is calculated as a conventional LEED state 34 with $\mathbf{k}_{\|}=0$ to describe the normally incident electron beam in the experimental setup. At kinetic energies of the order of $\mathrm{keV}$, however, the $\mathbf{k}_{\|}$ dependence turns out to be weak. Furthermore, at high kinetic energies, multiple-scattering effects are small and may be neglected for convenience.

The (paramagnetic) LDA potential for $\mathrm{Ni}$ is determined by a self-consistent tight-binding linear muffintin orbitals (LMTO) calculation. 35 The $3 d, 4 s$, and $4 p$ valence orbitals $|i L \sigma\rangle$ are taken to be the muffin-tin orbitals (MTO's). The four-fold degenerate $2 p_{3 / 2}$ core state is obtained from the LDA core potential by solving the radial Dirac equation numerically. Its (relativistically) large component is decomposed into a (coherent) sum of Pauli spinors $\left|2 \mathrm{p}, \sigma_{c}\right\rangle$ with $\sigma_{c}=\uparrow, \downarrow$.

Note that the Green function in Eq. (1) generally depends on four quantum numbers $L_{1}-L_{4}$. In fact, for the present case each term in the sum gives a significant contribution to $I_{\sigma_{c} \sigma_{i}}$. The usual characterization of the final state with two quantum numbers $(d-d, s-d$, etc. $)$ is no longer valid if correlations are included. The orbital character may change by electron scattering.

Neglecting electron correlations altogether and assuming the matrix elements to be constant, Eq. (1) reduces to the Lander model: In this case the intensity $I_{\sigma_{i}}$ is given by the following sum of (singlet and triplet) selfconvolutions:

$$
\begin{aligned}
I_{\sigma_{i}} & \propto \sum_{L_{1} L_{2}} \int d E^{\prime} \widetilde{\rho}_{L_{1} \sigma_{i}}\left(E^{\prime}\right) \widetilde{\rho}_{L_{2}-\sigma_{i}}\left(E-E^{\prime}\right) \\
& +\sum_{L_{1} L_{2}}^{\prime} \int d E^{\prime} \widetilde{\rho}_{L_{1} \sigma_{i}}\left(E^{\prime}\right) \widetilde{\rho}_{L_{2} \sigma_{i}}\left(E-E^{\prime}\right) .
\end{aligned}
$$

Here $\widetilde{\rho}_{L \sigma}(E)=(1-f(E)) \rho_{L \sigma}(E)$ is the unoccupied part of the $L$-resolved and spin-dependent density of states (DOS) $\rho_{L \sigma}(E)$ where $f(E)$ is the Fermi function. The prime in the second sum in Eq. (5) excludes the term $L_{1}=L_{2}, \sigma_{c}=\sigma_{i}$, which is forbidden by the Pauli principle. Note that lattice symmetries require the on-site $\left(i=i^{\prime}\right)$ but off-diagonal $\left(L \neq L^{\prime}\right)$ DOS $\rho_{L L^{\prime} \sigma}(E)=$ $(-1 / \pi) \operatorname{Im}\left\langle\left\langle c_{i L \sigma} ; c_{i L^{\prime} \sigma}^{\dagger}\right\rangle\right\rangle_{E}$ to vanish identically. This is a consequence of the choice of the (non-orthogonal) basis set $|i L \sigma\rangle$.

To estimate the significance of correlation effects, the two-particle Green function in Eq. (1) is calculated for a nine-band Hubbard-type model $H=H_{\mathrm{LDA}}+H_{\mathrm{int}}-\Delta H$ including $4 s, 4 p$ orbitals and correlated $3 d$ orbitals:

$$
\begin{aligned}
H & =\sum_{i i^{\prime} L L^{\prime} \sigma} T_{i i^{\prime}}^{L L^{\prime}} c_{i L \sigma}^{\dagger} c_{i^{\prime} L^{\prime} \sigma} \\
& +\frac{1}{2} \sum_{i \sigma \sigma^{\prime}} \sum_{m_{1} \ldots m_{4}} U_{m_{1} m_{2} m_{4} m_{3}} c_{i m_{1} \sigma}^{\dagger} c_{i m_{2} \sigma^{\prime}}^{\dagger} c_{i m_{3} \sigma^{\prime}} c_{i m_{4} \sigma} \\
& -\Delta H .
\end{aligned}
$$

The hopping parameters $T_{i i^{\prime}}^{L L^{\prime}}$ of the one-particle term $H_{\mathrm{LDA}}$ (and also the overlap $S_{i i^{\prime}}^{L L^{\prime}}$ parameters) are ob- 
tained from a Slater-Kester fit to the paramagnetic LDA band structure for Ni. 32 Opposed to photoemission spectroscopy, this comparatively simple tight-binding parameterization appears to be sufficient in the case of APS since the two-particle spectrum does not crucially depend on the details of the one-particle DOS.

The on-site interaction among the $3 d$ electrons is described by the second term $H_{\text {int }}$. The Coulomb matrix depends on four orbital indices, $U_{m_{1} m_{2} m_{3} m_{4}}$, referring to the MTO's for $l=2$. Using atomic symmetries the interaction parameters can essentially be expressed in terms of two parameters $U$ and $J 32$ Interactions involving delocalized $s$ and $p$ states are assumed to be sufficiently accounted for within the LDA. For the $d$ states, however, there is the well-known double-counting problem: The interactions are counted twice, once in $H_{\mathrm{LDA}}$ (i. e. on a mean-field level) and once more in $H_{\text {int }}$. To avoid this double counting, a third term $\Delta H$ has been introdped by which the Hartree-Fock part of $H_{\text {int }}$ is subtracted 32,36

The Hamiltonian $H$ constitutes an involved manybody problem. Due to the low density of $3 d$ holes in the case of $\mathrm{Ni}$, however, it appears to bereasonable to employ the so-called ladder approximation 29 which extrapolates from the exact (Cini-Sawatzky) solution for the limit of the completely filled band15.16 For finite hole densities the ladder approximation gives the two-particle Green function $\left\langle\left\langle c c ; c^{\dagger} c^{\dagger}\right\rangle\right\rangle$ as a functional of the one-particle Green function ("direct correlations"). The one-particle Green function of the type $\left\langle\left\langle c ; c^{\dagger}\right\rangle\right\rangle$ corresponds to the (inverse) photoemission spectrum which itself is renormalized by $H_{\text {int }}$ ("indirect correlations"). It is calculated self-consistently within second-order perturbation theory (SOPT) around the Hartree-Fock solution. 32 For a moderate $U \sim \Delta$ and a low hole density, a perturbational approach can be justified 36 A resummation of higher-order diagrams isimportant to describe bound states ("Ni $6 \mathrm{eV}$ satellite")B7 which, however, are relevant for AES only. The numerical values for the direct and exchange interaction, $U=2.47 \mathrm{eV}$ and $J=0.5 \mathrm{eV}$, are taken from Ref. 32 where they have been fitted to the ground-state magnetic properties of Ni. Since spin-wave excitations are neglected in the approach, the calculated Curie temperature $T_{\mathrm{C}}=1655 \mathrm{~K}$ is about a factor 2.6 too high compared with the experiment. Using reduced temperatures $T / T_{\mathrm{C}}$, however, the temperature trend of the magnetization is well reproduced. 32

\section{RESULTS AND DISCUSSION}

The solid lines in Fig. 1 (left) show the spectra as calculated from Eq. (1) using the ladder approximation. To account for apparatus broadening, the results have been convoluted with a Gaussian of width $\sigma=0.6 \mathrm{eV}$ (see Ref. 23). The calculated data are shifted by $852.3 \mathrm{eV}$ such that onset of the unbroadened spectrum for $T / T_{\mathrm{C}}=0.16$ coincides with the maximum of $L_{\mathrm{III}}$ emission in the experiment (dotted line).
What are the signatures of electron correlations? To estimate first the effect of the direct interaction between the two additional final-state electrons, Fig. 11 (right) also displays the results of the self-convolution model for comparison (still including matrix elements as well as the fully interacting one-particle DOS). Fig. 11 and also a more detailed inspection show that the secondary peak at $E \approx 859 \mathrm{eV}$ is not affected by correlations at all. This is consistent with observed temperature independence of the peak and with the fact that the DOS has mainly $s-p$ character at the discontinuity deriving from the $L_{7}$ critical point. The maximum of the secondary peak is therefore used as a reference to normalize the measured spectra for each $T$. Looking at the results of the ladder approximation, the overall agreement with the measurements is rather satisfying. Except for the lowest temperature the intensity, the spin splitting and the spin asymmetry of the main peak are well reproduced and, consistent with the experiment, a negligibly small intensity asymmetry and spin splitting is predicted for the secondary peak. Switching off the direct correlations (Fig. 1, right) results in a strong overestimation of the main peak structure. Within the Cini-Sawatzky theory this has a plausible qualitative explanation: For low hole density the main effect of the direct correlations is known to transfer spectral weight to lower energies inaccessible to APS. This weight shows up again in the Auger spectrum. Recall that in fact a considerable weight transfer is seen in AES10,26 and that hypothetically for $U \mapsto \infty$ all weight would be taken by a satellite pplit off at the lower boundary of the Auger spectrum.15.16

The indirect correlations manifest themselves as a renormalization of the one-particle DOS. Thus, in first place they are responsible for the correct temperature dependence of the intensity asymmetry of the main peak in the AP spectrum. In particular, the indirect correlations result in a narrowing of the DOS, which near the Fermi energy $E_{\mathrm{F}}$ is given by the (almost spin independent) quasi-particle weight $z \approx 0.88<1$, and in an intrinsic lifetime broadening $\Gamma \propto\left(E-E_{\mathrm{F}}\right)^{2}, T^{2}$. For $T=0$ the latter turns opt to be smaller than $\Gamma \approx 0.01 \mathrm{eV}$ for $E-E_{\mathrm{F}}<1 \mathrm{eV} .32$ The discrepancy between experiment and theory for $T / T_{\mathrm{C}}=0.16$ is possibly due to an underestimation of $\Gamma$ within the SOPT: With decreasing $T$ the increase of the exchange splitting leads to the appearance of a strong peak just above $E_{\mathrm{F}}$ in the minority DOS. A larger lifetime broadening of this peak would imply a less pronounced feature in the differential AP spectrum - mainly in the minority channel.

Setting $M_{L_{1} L_{2}}^{\sigma_{c} \sigma_{i}}\left(\mathbf{k}_{\|}, E\right)= \pm 1=$ const for $L_{1} \geq L_{2}$ or $L_{1}<L_{2}$, respectively, (see below) and comparing with the results of the full theory, demonstrates the importance of the transition-matrix elements. Their energy dependence (via $\left|\mathbf{k}_{\|} E \sigma_{i}\right\rangle$ ) is weak over a few eV at energies of the order of $\mathrm{keV}$ and cannot explain the difference between (a) and (b) in Fig. 2. The main difference is rather a consequence of the fact that the radial $2 p$ core wave function has a stronger overlap with the (more lo- 


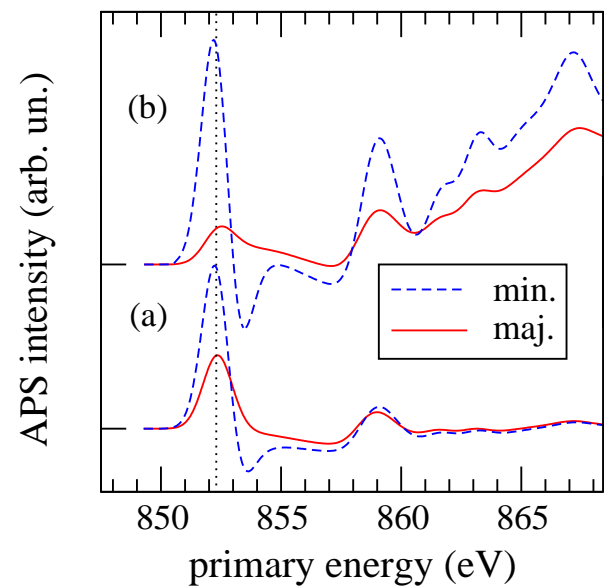

FIG. 2: Ni AP spectrum for $T=0$. (a) full theory. (b) as (a) but matrix elements taken to be constant (see text).

calized) $3 d$ as compared to the (more delocalized) $4 s / 4 p$ radial wave functions. This implies a suppression of the $s-p$ contributions to the orbital sum in Eq. (11) as already found by Ebert and Popescu. 23 The features above $E=860 \mathrm{eV}$ originate from additional discontinuities of the $s$-p-like DOS (as for the peak at $E \approx 859 \mathrm{eV}$ ).

For $T<T_{\mathrm{C}}$ the spin asymmetry of the spectrum is mainly due to the spin dependence of the Green function in Eq. (11). If the calculation of the matrix elements (14) starts from the spin-polarized L(S)DA potential, an additional spin asymmetry is observed resulting from the spin dependence of the states in Eq. (44). This, however, is small and has practically no influence on the results. On the other hand, Fig. 2 shows a strong suppression of the intensity asymmetry at high energies when taking matrix elements into account. This effect is controlled by the symmetry of the matrix $M_{L_{1} L_{2}} \equiv M_{L_{1} L_{2}}^{\sigma_{c} \sigma_{i}}\left(\mathbf{k}_{\|}, E\right)$. In the antisymmetric case, $M_{L_{1} L_{2}}=-M_{L_{2} L_{1}}\left(L_{1} \neq L_{2}\right)$, there is a maximum spin asymmetry (Fig. 2) while, even for a ferromagnet, there is no spin asymmetry at all for the symmetric case. The results of the full calculation along Eq. (4) are neither fully symmetric nor antisymmetric with respect to $L_{1}, L_{2}$.

\section{SUMMARY}

In conclusion, the AP line shape of a typical ferromagnetic $3 d$ transition metal results from a rather com- plex interplay of different factors. The present study has shown that a quantitative theoretical analysis of the temperature- and spin-dependent spectrum must be based on three ingredients at least:

(i) "indirect" correlations: A realistic Hubbard-type model including $s-p$ like states is a proper starting point to describe the magnetism and the temperaturedependent renormalization of the one-particle DOS.

(ii) "direct" correlations: While $s-p$ derived features at higher energies appear to be sensitive to the geometrical structure only, the main peak is strongly affected by the direct interaction between the two additional final-state electrons. Consistent with the Cini-Sawatzky model, the dominant effect is a considerable spectral-weight transfer to energies below the threshold.

(iii) matrix elements: Spin-resolved APS cannot be described theoretically without calculating orbitaldependent matrix elements. The spin asymmetry is mainly determined by their transformation behavior under exchange of the orbital quantum numbers $L_{1} \leftrightarrow L_{2}$.

An open question concerns the importance of core-hole effects in APS. Future work shall be concerned with the scattering at the core-hole potential in the final state and shall include the edge effects 38 known from the studies of simple metals. A generalized ladder approximation including the valence-core interaction has been proposed in Ref. 20. Previous work 192221 has shown that one should not expect the effects due the scattering at the core-hole potential to be strong for the case of Ni. However, corehole effects will become more important for systems with a smaller $3 d$ occupancy. For Co and Fe one also expects stronger effects of $d-d$ correlations. This work has shown that these $d-d$ correlations cannot be neglected even for a system with low $d$-hole density such as $\mathrm{Ni}$ and that deconvolution techniques to extract the unoccupied local DOS have to be questioned seriously.

\section{Acknowlegdements}

Support of this work by the DFG (NO 158/5) and by the BMBF (no. 05605MPA0) is greatfully acknowledged.
* email: michael.potthoff@physik.hu-berlin.de

1 R. L. Park and J. E. Houston, J. Vac. Sci. Technol. 11, 1 (1974).

2 K. Wandelt and G. Ertl, J. Phys. F 6, 1607 (1976).

3 V. Dose and R. Reusing, Solid State Commun. 48, 683 (1983).
${ }^{4}$ C. J. Powell, N. E. Erickson, and D. E. Ramaker, Phys. Scr. T41, 175 (1992).

5 J. Kirschner, Solid State Commun. 49, 39 (1984).

${ }^{6}$ K. Ertl, M. Vonbank, V. Dose, and J. Noffke, Solid State Commun. 88, 557 (1993).

7 T. Detzel, M. Vonbank, M. Donath, and V. Dose, J. Magn. 
Magn. Mat. 147, L1 (1995).

8 G. Rangelow, H. D. Kang, J. Reinmuth, and M. Donath, Phys. Rev. B 61, 549 (2000).

9 H. D. Kang, G. Rangelow, J. Reinmuth, and M. Donath, Surf. Sci. 454-456, 865 (2000).

10 D. E. Ramaker, Crit. Rev. Solid State Mater. 17, 211 (1991).

11 J. J. Lander, Phys. Rev. 91, 1382 (1953).

12 V. Dose and T. Fauster, Appl. Phys. 20, 1 (1979).

13 S. W. Schulz, K. T. Schleicher, D. M. Rück, and H. U. Chun, J. Vac. Sci. Technol. A 2, 822 (1984).

14 C. J. Powell, Phys. Rev. Lett. 30, 1179 (1973).

15 M. Cini, Solid State Commun. 24, 681 (1977).

16 G. A. Sawatzky, Phys. Rev. Lett. 39, 504 (1977).

17 M. Kotrla and V. Drchal, J. Phys.: Condens. Matter 1, 4783 (1992).

18 W. Nolting, G. Geipel, and K. Ertl, Phys. Rev. B 45, 5790 (1992).

19 M. Potthoff, J. Braun, W. Nolting, and G. Borstel, J. Phys.: Condens. Matter 5, 6879 (1993).

20 M. Potthoff, J. Braun, and G. Borstel, Z. Phys. B 95, 207 (1994).

21 M. Potthoff, J. Braun, G. Borstel, and W. Nolting, J. Electron Spectrosc. Rel. Phen. 72, 163 (1995).

22 G. Hörmandinger, P. Weinberger, P. Marksteiner, and J. Redinger, Phys. Rev. B 38, 1040 (1988).

23 H. Ebert and V. Popescu, Phys. Rev. B 56, 12884 (1997).
24 J. Reinmuth, F. Passek, V. N. Petrov, M. Donath, V. Popescu, and H. Ebert, Phys. Rev. B 56, 12893 (1997).

${ }^{25}$ G. Tréglia, M. C. Desjonquères, F. Ducastelle, and D. Spanjaard, J. Phys. C 14, 4347 (1981).

26 P. A. Bennett, J. C. Fuggle, F. U. Hillebrecht, A. Lenselink, and G. A. Sawatzky, Phys. Rev. B 27, 2194 (1983).

27 M. B. Stearns (Springer, Berlin, 1984), vol. 19a of Group III, chap. Magnetic Properties of Metals.

28 M. Donath, Appl. Phys. A 49, 351 (1989).

29 M. Cini, Surf. Sci. 87, 483 (1979).

30 D. N. Zubarev, Sov. Phys. Uspekhi 3, 320 (1960).

31 J. Dorantes-Dávila, A. Vega, and G. M. Pastor, Phys. Rev. B 47, 12995 (1993).

32 T. Wegner, M. Potthoff, and W. Nolting, Phys. Rev. B 61, 1386 (2000).

33 T. Schlathölter, thesis, Universität Osnabrück (2000).

34 J. B. Pendry, Low Energy Electron Diffraction (Academic Press, London, 1974).

35 O. Andersen and O. Jepsen, Phys. Rev. Lett. 53, 2571 (1984).

36 M. M. Steiner, R. C. Albers, and L. J. Sham, Phys. Rev. B 45, 13272 (1992).

37 A. Liebsch, Phys. Rev. B 23, 5203 (1981).

38 P. Nozières and C. T. DeDominicis, Phys. Rev. 178, 1097 (1969). 